\title{
Developing near-infrared quantum-dot light-emitting diodes to mimic synaptic plasticity
}

\author{
Shuangyi Zhao ${ }^{1}$, Yue Wang ${ }^{1}$, Wen Huang ${ }^{1}$, Hao Jin ${ }^{1}$, Peiwen Huang ${ }^{1}$, Hu Wang ${ }^{1}$, Kun Wang ${ }^{1}$, \\ Dongsheng $\mathrm{Li}^{1}$, Mingsheng $\mathrm{Xu}^{2}$, Deren Yang ${ }^{1}$ and Xiaodong $\mathrm{Pi}^{1 *}$
}

\begin{abstract}
The quantum-dot light-emitting diodes (QLEDs) that emit near-infrared (NIR) light may be important optoelectronic synaptic devices for the realization of artificial neural networks with complete optoelectronic integration. To improve the performance of NIR QLEDs, we take advantage of their low-energy light emission to explore the use of poly(3-hexylthiophene) (P3HT) as the hole transport layer (HTL). P3HT has one of the highest hole mobilities among organic semiconductors and essentially does not absorb NIR light. The usage of P3HT as the HTL indeed significantly mitigates the imbalance of carrier injection in NIR QLEDs. With the additional incorporation of an interlayer of poly [9,9-bis( $3^{\prime}$-( $N, N$-dimethylamino)propyl)-2,7-flourene $]$ alt-2,7-(9,9-dioctylfluorene)], P3HT obviously improves the performance of NIR QLEDs. As electroluminescent synaptic devices, these NIR QLEDs exhibit important synaptic functionalities such as short- and long-term plasticity, and may be employed for image recognition.
\end{abstract}

Keywords: quantum-dot light-emitting diodes, near-infrared, synaptic devices, poly(3-hexylthiophene)

\section{INTRODUCTION}

Neuromorphic computing has drawn increasing attention because it is more energy-efficient and intelligent than conventional von Neumann computing, which faces imminent physical limits and explosively increased energy consumption [1-3]. To develop neuromorphic computing, artificial neural networks based on high-performance synaptic devices must be produced [2,4-12]. Recent research in neuroscience on optogenetics has indicated that the functionality of neuromorphic computing may be significantly enhanced by incorporating light into an artificial neural network [13-17]. Hence, optoelectronic synaptic devices have significant potential. Considerable effort has been expended to fabricate optoelectronic synaptic devices that are stimulated optically and produce an electrical output [18-22]. Furthermore, the devices that are electrically stimulated and produce an optical output have recently been demonstrated [23]. Combining these features would enable bidirectional conversion between optical and electrical signals, facilitating the realization of neuromorphic optoelectronics [24], which holds considerable promise for high-performance computing because of the combination of the brain-like low-energy processing capacity with the speed and bandwidth of the photonic devices [24].

Near-infrared (NIR) light is a good choice with respect to the optical output of an optoelectronic synaptic device because the loss of NIR light is usually low during transmission through many technologically important materials $[25,26]$. Hence, high-performance quantum-dot light-emitting diodes (QLEDs) that emit NIR light are worth investigating as optoelectronic synaptic devices. However, NIR QLEDs have serious issues, such as the carrier injection imbalance, which should be tackled $[27,28]$. The carrier injection imbalance mainly occurs because the hole mobility of the routinely used organic hole-transport layer (HTL) for a QLED is much lower than the electron mobility of the popular electron-transport layer of zinc oxide $(\mathrm{ZnO})$ nanocrystals $(\sim 1.8 \times$ $10^{-3} \mathrm{~cm}^{2} \mathrm{~V}^{-1} \mathrm{~s}^{-1}$ ) [29-31]. For example, an HTL of poly ( $N, N^{\prime}$-bis(3-methylphenyl)- $N, N^{\prime}$-diphenylbenzidine), also known as poly-TPD, has a hole mobility of only $\sim 1.0 \times$ $10^{-4} \mathrm{~cm}^{2} \mathrm{~V}^{-1} \mathrm{~s}^{-1}$ [29]. Poly(3-hexylthiophene) (P3HT) is one of the most extensively used organic semiconductors. It has a high hole mobility of $\sim 1.0 \times 10^{-3} \mathrm{~cm}^{2} \mathrm{~V}^{-1} \mathrm{~s}^{-1}$ $[32,33]$, which is quite comparable to the electron mo-

\footnotetext{
${ }^{1}$ State Key Laboratory of Silicon Materials \& School of Materials Science and Engineering, Zhejiang University, Hangzhou 310027, China

${ }^{2}$ College of Information Science and Electronic Engineering, Zhejiang University, Hangzhou 310027, China

* Corresponding author (email: xdpi@zju.edu.cn)
} 
bility of an electron-transport layer of $\mathrm{ZnO}$ nanocrystals. In addition, P3HT is very suitable for the HTL of NIR QLEDs because it does not absorb NIR light. For NIR QLEDs, P3HT should significantly mitigate the carrier injection imbalance without deteriorating the light extraction. Therefore, it would be interesting to harness the remarkable electronic and optical properties of P3HT for NIR QLEDs.

In this work, we investigate the incorporation of a P3HT HTL into NIR QLEDs based on silicon (Si) quantum dots (QDs), which have efficient luminescence and robust photostability [34]. The thickness of the P3HT HTL is first optimized. An interlayer of poly[9,9-bis(3'(N,N-dimethylamino)propyl)-2,7-flourene]-alt-2,7-(9,9dioctylfluorene)] (PFN) [35] is then added between the P3HT and the Si QDs to further enhance the device performance, which is better than that of NIR QLEDs with a traditional poly-TPD HTL. We finally demonstrate the use of NIR QLEDs with a P3HT HTL as optoelectronic synaptic devices. Important synaptic functionalities such as short-term plasticity $[22,36]$ and long-term plasticity (LTP) $[17,37]$ are realized. Simple image recognition could be carried out with these optoelectronic synaptic devices.

\section{EXPERIMENTAL SECTION}

\section{Materials and devices}

Freestanding Si QDs were synthesized in a nonthermal plasma system $[38,39]$. Their surfaces were subsequently hydrosilylated with 1-heptylene. We then dispersed the hydrosilylated Si QDs in toluene to obtain a solution of Si QDs with a concentration of $15 \mathrm{mg} \mathrm{mL}^{-1}$. A layer of poly(3,4-ethylenedioxythiophene)/polystyrene sulfonate (PEDOT:PSS), which had a thickness of $\sim 20 \mathrm{~nm}$, was produced by spin-coating a PEDOT:PSS solution onto clean indium tin oxide (ITO)/glass substrates at $4000 \mathrm{rpm}$ for $60 \mathrm{~s}$. Then the substrates were baked on a hotplate at $130^{\circ} \mathrm{C}$ for $20 \mathrm{~min}$. A P3HT solution was obtained by dispersing $\mathrm{P} 3 \mathrm{HT}$ in chlorobenzene. It had a concentration of $10-25 \mathrm{mg} \mathrm{mL}^{-1}$, allowing us to study the effect of the thickness of the resulting P3HT layer on device performance. After being stirred overnight, the $\mathrm{P} 3 \mathrm{HT}$ solution was spin-coated onto the PEDOT:PSS. Solvent annealing and thermal annealing were carried out immediately after the spin-coating [40]. Solutions with $2 \mathrm{mg} \mathrm{mL}^{-1}$ of PFN, $10 \mathrm{mg} \mathrm{mL}^{-1}$ of poly-TPD, and $25 \mathrm{mg} \mathrm{mL}^{-1}$ of $\mathrm{ZnO}$ were obtained by dispersing them in methanol, chlorobenzene, and ethanol, respectively. Then, the PFN, Si QD, poly-TPD, and ZnO solutions
$[35,41]$ were spin-coated consecutively at $2000 \mathrm{rpm}$ for $50 \mathrm{~s}$ to form an $\sim 5$-nm-thick PFN layer, a 30-nm-thick SiQD layer, a $20-\mathrm{nm}$-thick poly-TPD layer, and a $50-\mathrm{nm}$ thick $\mathrm{ZnO}$ layer, respectively. After each spin-coating, the samples were baked at $110^{\circ} \mathrm{C}$ for $20 \mathrm{~min}$. Note that the thicknesses of the PEDOT:PSS, poly-TPD, PFN, Si-QD and $\mathrm{ZnO}$ layers were chosen based on previous highperformance device structures $[29,35,41]$. Finally, a 150 $\mathrm{nm}$-thick layer of Ag was deposited by thermal evaporation. The area of each device was $4 \mathrm{~mm}^{2}$.

\section{Characterization}

The Si QDs were characterized with a $100 \mathrm{kV}$ transmission electron microscope (TEM; JEM-2100F, JEOL). A photoluminescence (PL) characterization system (FLS920, Edinburgh Instruments) was used to measure the light emitted from the Si QDs and NIR QLEDs. An atomic force microscope (Multimode-8, Bruker Dimension Edge) was employed to study the morphology of the thin-film layers. A Keithley 2400 source meter was used to obtain the $J-V$ curves of the LEDs. A Newport 1931-C power meter coupled with a Newport 818-UV photodetector was employed to measure the optical power of the LEDs [23]. The functionality of the synaptic devices was characterized using a homemade system, mainly comprising a pulse generator (DG4202 Rigol) and an oscilloscope (Wavesurfer 3024, Teledyne) [23].

\section{Simulation}

An image of an eagle was used for the simulation of image recognition. The image was divided into 1960 squares. As more squares were recognized, the definition of the image increased. Hence, the image looked clearer. It was assumed that the maximum optical power evoked by 30 electrical spikes $\left(P_{30}\right)$ corresponded to all squares being recognized. The number of recognized squares was obtained by considering the ratio of the maximum optical power evoked by $i(i=2,5,10,20,30)$ electrical spikes $\left(P_{i}\right)$ to that evoked by 30 electrical spikes $\left(P_{30}\right)$, i.e., $P_{i} / P_{30}$. As more electrical spikes were used, $P_{i} / P_{30}$ became larger, leading to a clearer image.

\section{RESULTS AND DISCUSSION}

Fig. 1a shows a low-resolution TEM image of Si QDs that can emit NIR light. The crystallinity of the Si QDs is evidenced by the selected-area electron diffraction image (the inset of Fig. 1a) and the single-QD high-resolution TEM image (Fig. 1b). A statistical analysis shows that the mean size of the Si QDs is $\sim 4.2 \mathrm{~nm}$ (Fig. 1c). Fig. 1d displays the PL spectra of the Si QDs. There is a peak at 

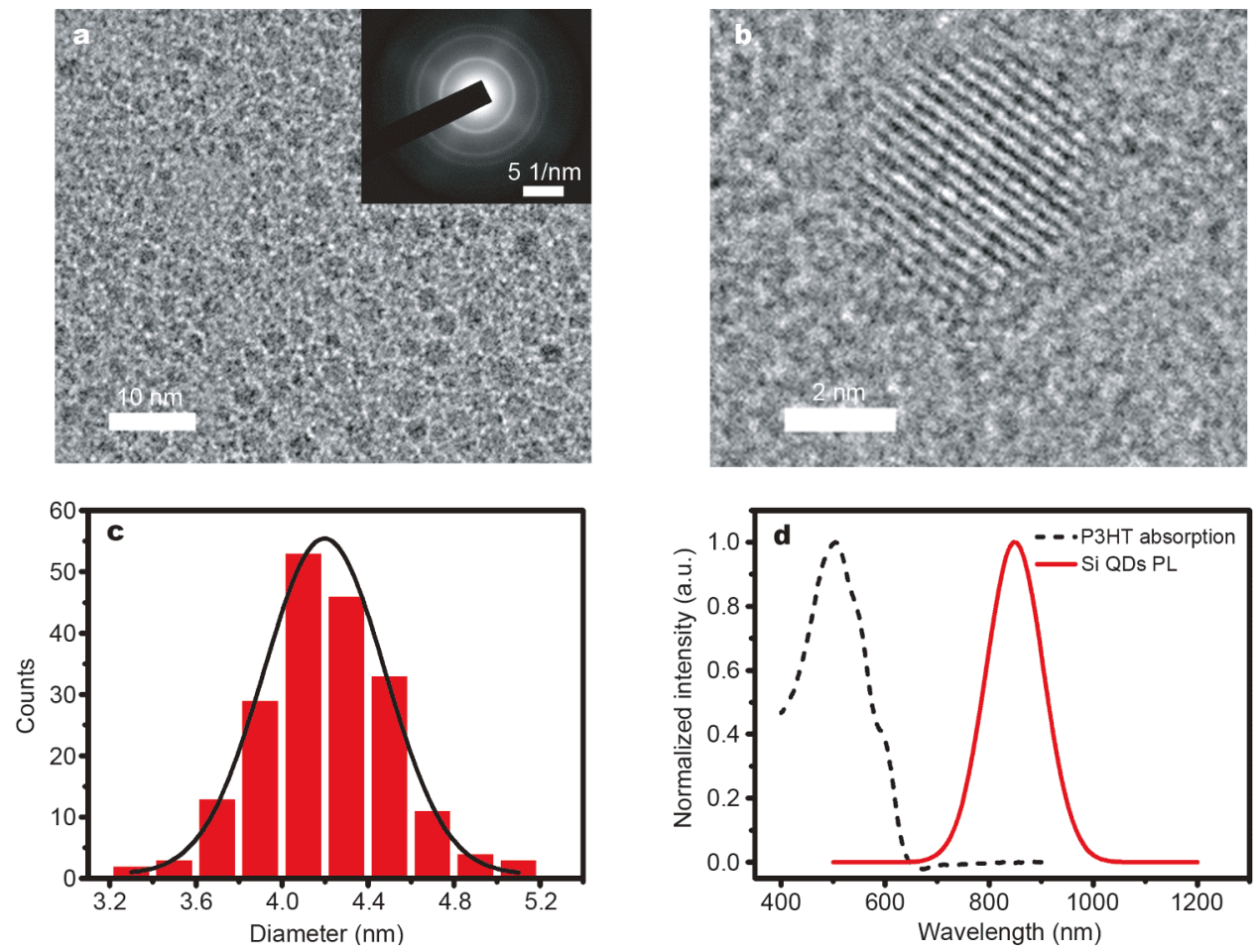

Figure 1 Characterization of Si QDs. (a) Low-resolution TEM image of Si QDs. The inset shows an electron diffraction photograph of the selected area. (b) High-resolution TEM image of a Si QD. (c) Size distribution with a log-normal fit for Si QDs. The mean size of the Si QDs is $\sim 4.2 \mathrm{~nm}$. (d) UV-visible optical absorption spectrum of P3HT and PL spectrum of Si QDs.

$\sim 850 \mathrm{~nm}$. It is clear that this NIR light emitted from the $\mathrm{Si}$ QDs is outside the spectral region (wavelengths from $\sim 400$ to $650 \mathrm{~nm}$ ) for the absorption of P3HT (Fig. 1d). Hence, P3HT will not affect the NIR light emitted by Si QDs due to the absorption in NIR QLEDs based on Si QDs. This is verified by further examining the ultraviolet (UV)-visible absorption and PL spectra of the hybrid structure of P3HT and Si QDs (Fig. S1).

By using P3HT as the HTL, we fabricated NIR QLEDs based on Si QDs with a layered structure of glass/ITO/ PEDOT:PSS/P3HT/Si-QDs/ZnO/Ag, as schematically shown in Fig. 2a. The ITO layer on the glass substrate and the Ag layer are the anode and cathode, respectively. The PEDOT:PSS layer is used to facilitate the injection of holes [41]. The $\mathrm{ZnO}$ layer acts as the electron-transport layer, which has been adopted in most high-performance QLEDs [29]. Fig. 2b shows the flat-band energy-level diagram of a NIR QLED based on Si QDs. The conduction band minimum (CBM) and valence band maximum (VBM) of Si QDs are located at -4.0 and $-5.4 \mathrm{eV}$, respectively [42]. The small-step cascade alignment from the work function of ITO to the highest occupied molecular orbital (HOMO) of PEDOT, the HOMO of P3HT and the VBM of Si QDs ensures that holes are effectively injected into the Si-QD layer [43-45]. Similarly, the small-step cascade alignment from the work function of $\mathrm{Ag}$ to the CBM of $\mathrm{ZnO}$ [46], and the CBM of Si QDs facilitates the injection of electrons into the Si-QD layer. Holes and electrons injected into the Si-QD layer may radiatively recombine, giving rise to electroluminescence (EL).

In the current work, the effect of the thickness of P3HT layer on the device performance is evaluated. The thickness of the P3HT layer is controlled by the concentration of the P3HT solution during spin coating (Fig. S2). As shown in Fig. S3, as the concentration of the P3HT solution varies from 10 to $25 \mathrm{mg} \mathrm{mL}^{-1}$, the thickness of the P3HT layer changes from $\sim 50$ to $\sim 160 \mathrm{~nm}$. Current density $(J)$ versus voltage $(V)$ curves for NIR QLEDs based on Si QDs with different P3HT concentrations are shown in Fig. 2c. An increase of the P3HT concentration leads to a decrease of the current density, since the electrical resistance is larger for a thicker P3HT layer. Fig. 2d shows the EL of a NIR QLED fabricated with a P3HT concentration of $15 \mathrm{mg} \mathrm{mL}^{-1}$ at different driving voltages. Although the EL intensity increases as the driving voltage increases, the EL peak remains at $\sim 850 \mathrm{~nm}$. Since the peak of the PL for Si QDs is also at 


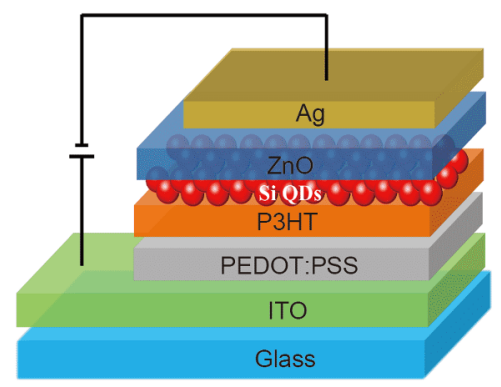

c

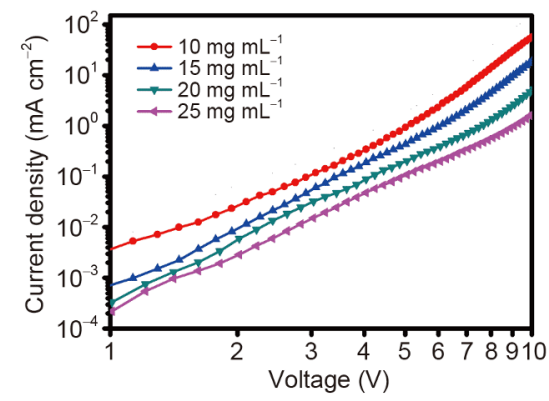

b

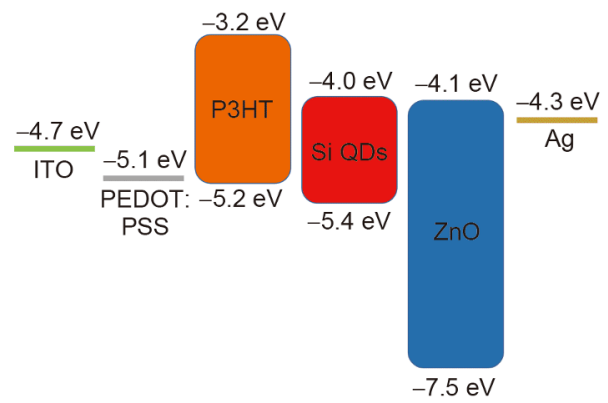

d

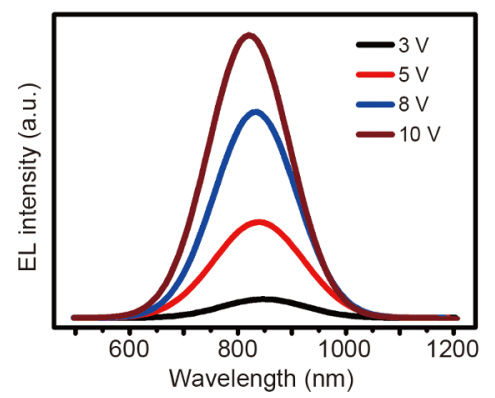

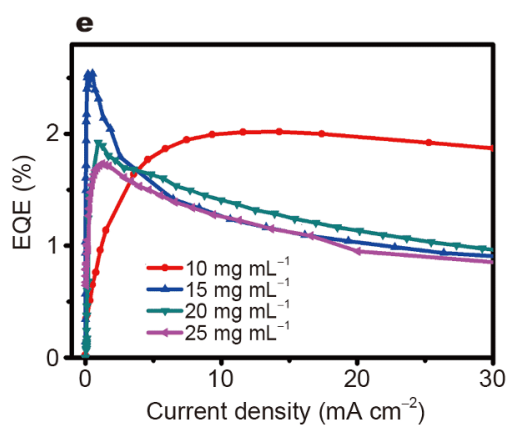
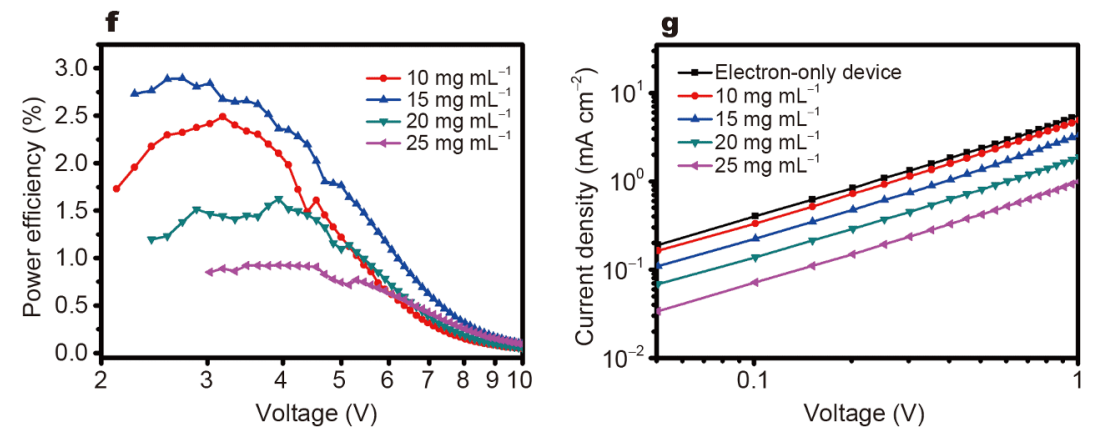

Figure 2 (a) Schematic diagram and (b) flat-band energy-level diagram of a NIR QLED based on Si QDs. (c) J- $V$ curves for the NIR QLEDs based on Si QDs with different P3HT concentrations. (d) EL spectra of a NIR QLED with a P3HT concentration of $15 \mathrm{mg} \mathrm{mL}^{-1}$ at different driving voltages. (e) EQE versus current density and (f) power efficiency versus voltage for the NIR QLEDs based on Si QDs with different P3HT concentrations. (g) J-V curves for an electron-only device and hole-only devices with different P3HT concentrations.

$\sim 850 \mathrm{~nm}$, the light emitted by these NIR QLEDs indeed originates from the Si QDs. Fig. 2e shows the external quantum efficiency (EQE) of NIR QLEDs based on $\mathrm{Si}$ QDs. The maximum EQE increases as the P3HT concentration increases from 10 to $15 \mathrm{mg} \mathrm{mL}^{-1}$, while it decreases with a further increase of the $\mathrm{P} 3 \mathrm{HT}$ concentration from 15 to $25 \mathrm{mg} \mathrm{mL}^{-1}$. The highest EQE of $2.6 \%$ was obtained with a P3HT concentration of $15 \mathrm{mg} \mathrm{mL}^{-1}$. In addition, a peak power efficiency of $2.8 \%$ was also obtained for NIR QLEDs with a P3HT concentration of $15 \mathrm{mg} \mathrm{mL}^{-1}$ (Fig. 2f). When the P3HT concentration is low, the resulting thin $\mathrm{P} 3 \mathrm{HT}$ layer may not be able to block the leakage of electrons injected into the Si-QD layer effectively. However, if the P3HT layer is too thick and has a very large resistance, then the injection of holes toward the Si-QD layer will be seriously weakened, which is demonstrated by the $J-V$ curves for hole-only devices fabricated with different $\mathrm{P} 3 \mathrm{HT}$ concentrations, as shown in Fig. 2g. A QLED for a P3HT concentration of $10 \mathrm{mg} \mathrm{mL}^{-1}$ shows the smallest roll-off. This may be because it has the weakest imbalance between electron injection and hole injection in the current work [27], as shown in Fig. 2g.

To improve the performance of NIR QLEDs based on Si QDs fabricated with the optimum P3HT concentration of $15 \mathrm{mg} \mathrm{mL}^{-1}$ (i.e., giving an optimum P3HT layer thickness of $\sim 80 \mathrm{~nm}$ ), we added an interlayer of PFN between the P3HT layer and Si-QD layer to mitigate the electron leakage from the Si-QD layer toward the anode. The resulting device structure is schematically illustrated 
in Fig. 3a. From the corresponding flat-band energy-level diagram of the device (Fig. 3b), we can see that the LUMO of PFN is higher than that of P3HT $(-2.8 \mathrm{eV}$ versus $-3.2 \mathrm{eV}$ ) [35]. A higher LUMO is better for blocking electron leakage from the Si-QD layer. Here, PFN was selected because it has already been effectively employed for the interfacial modification of optoelectronic devices [47]. Methanol, which is the solvent for PFN, is orthogonal with chlorobenzene, the solvent for P3HT, which hardly complicates device fabrication. In addition, the optical absorption of the P3HT layer hardly changes after the PFN layer is introduced (Fig. S4). Fig. S5 shows the morphologies of P3HT layers with and without a PFN interlayer. Both are of high quality with a rough- ness of only $\sim 0.7 \mathrm{~nm}$.

Fig. $3 c$ compares the $J-V$ curve of a device with PFN interlayer and that of a device only with P3HT. The addition of the PFN interlayer decreases the current density of the device. This is reasonable because the PFN interlayer introduces additional resistance for hole transport, as demonstrated by the decrease of the hole current when a PFN interlayer is used in a hole-only device (Fig. 3d). By fitting the $J-V$ results for electron-only and hole-only devices with the model of space charge limited transport (Fig. S6), the electron mobility of $\mathrm{ZnO}$ layer and the hole mobility of P3HT layer were calculated to be $1.5 \times 10^{-3}$ and $1.1 \times 10^{-3} \mathrm{~cm}^{2} \mathrm{~V}^{-1} \mathrm{~S}^{-1}$, respectively. Note that the hole transport (imbalance of carrier injection) of the device
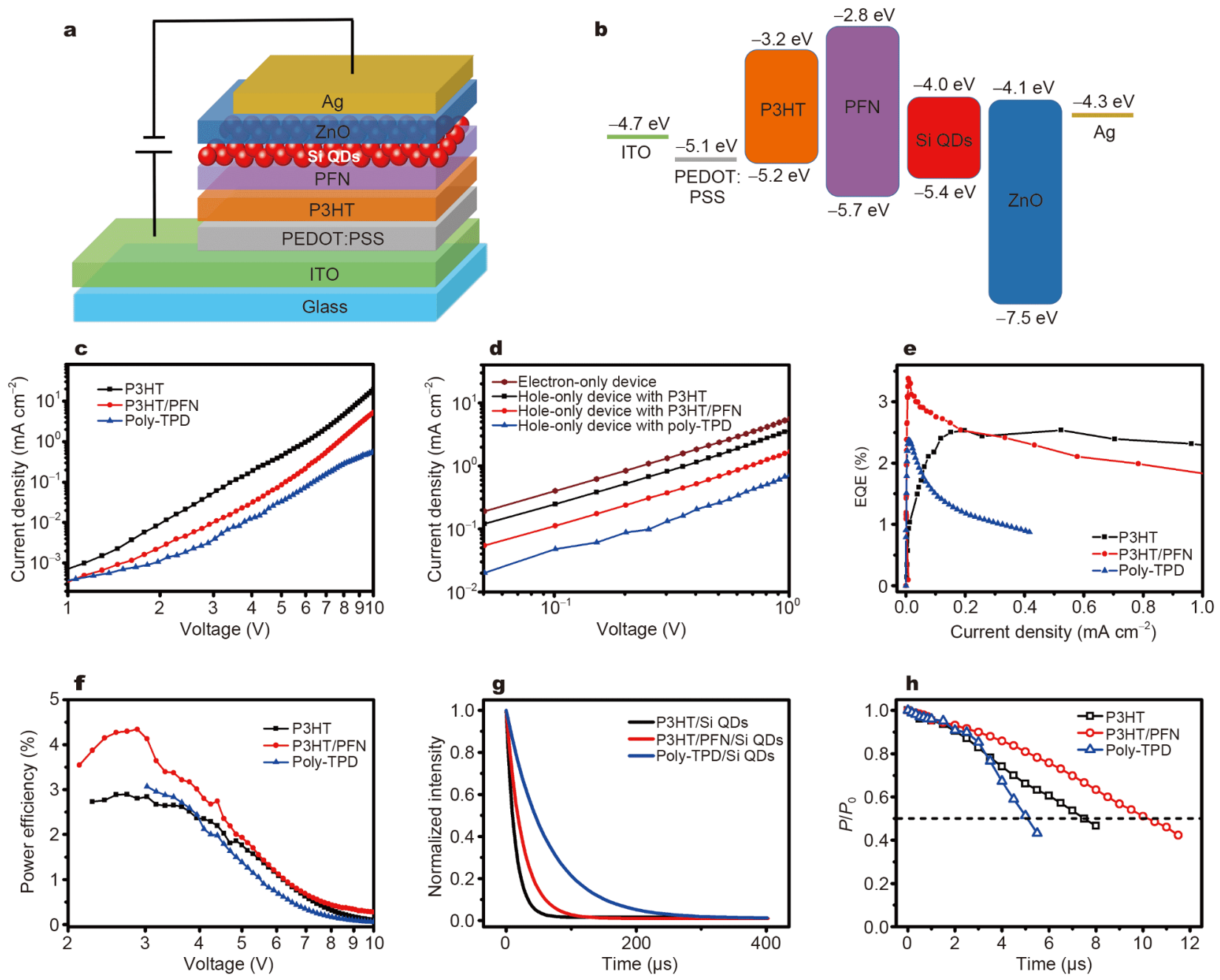

Figure 3 (a) Schematic diagram and (b) flat-band energy-level diagram of a NIR QLED based on Si QDs with a PFN interlayer. (c) $J-V$ curves for NIR QLEDs based on Si QDs with P3HT, P3HT/PFN and the traditional HTL of poly-TPD. (d) $J-V$ curves for an electron-only device and hole-only devices with P3HT, P3HT/PFN and poly-TPD. (e) EQE versus current density and (f) power efficiency versus voltage for NIR QLEDs based on Si QDs with P3HT, P3HT/PFN and poly-TPD. (g) PL decay curves of Si QDs with P3HT, P3HT/PFN and poly-TPD. (h) Stability of QLEDs with P3HT, $\mathrm{P} 3 \mathrm{HT} / \mathrm{PFN}$ and poly-TPD working at a driving voltage of $\sim 5 \mathrm{~V}$. 
with the PFN interlayer is better (less serious) than that of a device with the traditional HTL of poly-TPD, despite the increase in the device resistance due to the PFN interlayer (Fig. 3d). Hence, the current density of the device with the added PFN interlayer is still larger than that of a device with a traditional HTL of poly-TPD (Fig. 3b).

Fig. 3e shows that the addition of the PFN interlayer improves the maximum EQE of QLEDs with a P3HT HTL from $2.6 \%$ to $3.4 \%$, which is higher than that of QLEDs with the traditional HTL of poly-TPD (2.4\%). In addition, compared with QLEDs with a HTL of poly-TPD or P3HT, the QLED with a P3HT HTL and a PFN interlayer has a higher power efficiency of up to $4.4 \%$ (Fig. 3f). We compared the PL lifetimes of Si QDs in $\mathrm{P} 3 \mathrm{HT} / \mathrm{Si}-\mathrm{QDs}$, P3HT/PFN/Si-QDs, and poly-TPD/SiQDs, as shown in Fig. 3g. The addition of the PFN interlayer increases the PL lifetime from 21 to $33 \mu \mathrm{s}$, indicating that the $\mathrm{P} 3 \mathrm{HT}$ weakens exciton quenching $[48,49]$. This explains the PFN-interlayer-induced increase of the maximum EQE together with the electronblocking effect of the PFN interlayer. Note that the PL lifetime of Si QDs in poly-TPD/Si-QDs is the longest $(79 \mu \mathrm{s})$, implying the least amount of exciton quenching. However, the large carrier injection imbalance plays the dominant role in QLEDs with the traditional HTL of poly-TPD. Hence, the maximum EQE of the QLED with the traditional HTL of poly-TPD is lower than that of a QLED with a P3HT HTL and PFN interlayer (Fig. 3e). Fig. 3h shows the stability of the QLEDs with different structures at a driving voltage of $\sim 5 \mathrm{~V}$. The half-lifetimes $\left(t_{50}\right)$ for the QLEDs with the traditional HTL of poly-
TPD, a HTL of P3HT, and the P3HT/PFN combination without encapsulation are $\sim 5.0,7.5$, and $10.5 \mathrm{~h}$, respectively. The value of $\sim 10.5 \mathrm{~h}$ is higher than those of our previous electroluminescent synaptic devices [23] and comparable to those described in the literature [50]. The change in the stability is basically consistent with that for the power efficiency in the present work. It is expected that our QLEDs will be more stable after being encapsulated [27].

Electroluminescent synaptic devices with an electrical input and an optical output need to be fabricated in the optoelectronic integration of artificial neural systems. It has recently been demonstrated that QLEDs based on $\mathrm{Si}$ QDs can be used as electroluminescent synaptic devices with a low energy consumption [23]. Since a higher power efficiency helps reduce energy consumption [51], it is worth exploring the use of QLEDs with a P3HT HTL and a PFN interlayer as electroluminescent synaptic devices. Fig. 4a shows that an electroluminescent synaptic device can emit NIR light when it is stimulated by an electrical spike. The decay time of the EL (i.e., the time for the optical power to decrease from $90 \%$ to $10 \%$ of its original value) is $\sim 10 \mathrm{~ms}$, which is in the range of 1 $10^{4} \mathrm{~ms}$ that is typical for the decay time of a signal in a biological synapse $[52,53]$. When stimulated by two successive electrical spikes, the electroluminescent synaptic device exhibits clear paired-pulse facilitation (PPF) [9,53-56], which is the evidence for short-time plasticity [57-65], as shown in Fig. 4b. We calculated the PPF index using the ratio of the optical power evoked by the second electrical spike $\left(P_{2}\right)$ to that evoked by the first electrical
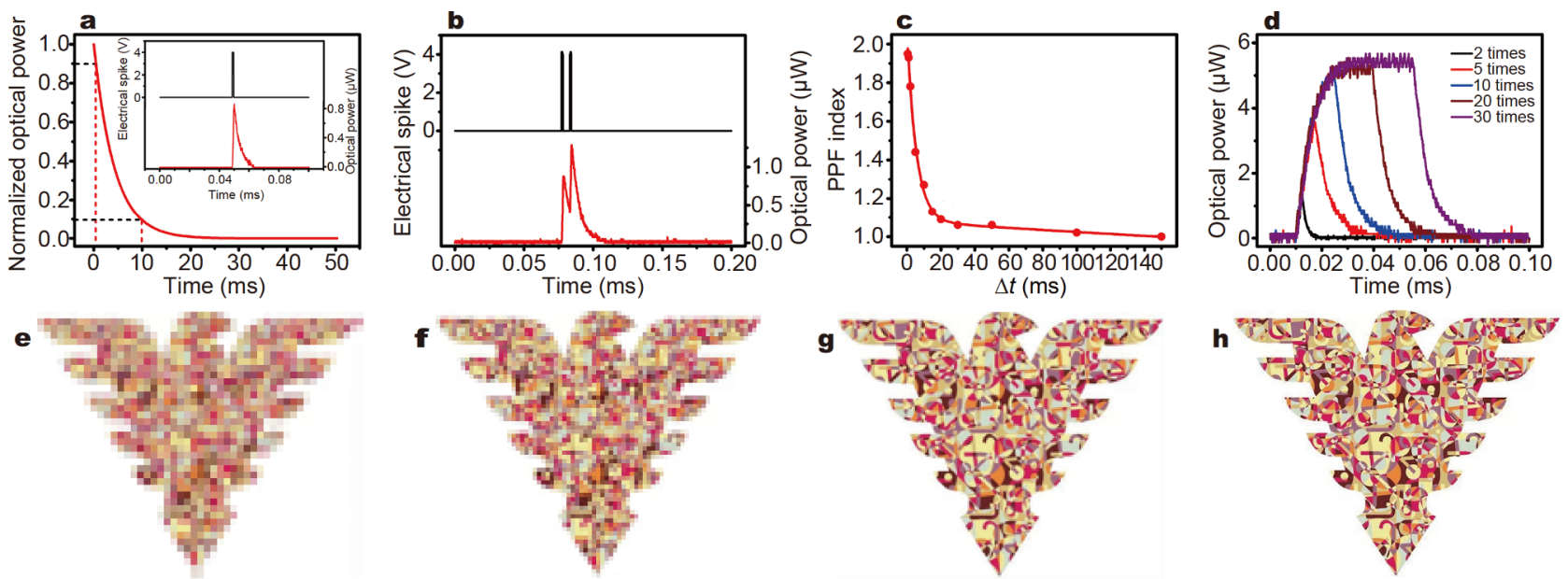

Figure 4 (a) Decay of the EL from a synaptic device stimulated by a $4 \mathrm{~V}$ electrical spike with a duration of 1 ms. The electrical spike and the corresponding optical power of the EL are shown in the inset. (b) Optical power of a synaptic device induced by two successive electrical spikes. (c) Dependence of the PPF index on $\Delta t$ for $4 \mathrm{~V}$ electrical spikes. (d) Optical power of a synaptic device stimulated by from two to thirty times of $4 \mathrm{~V}$ electrical spikes. Image recognition depictions for a synaptic device stimulated by $2,5,10$, and 20 successive electrical spikes are shown in (e-h). 
spike $\left(P_{1}\right)$. Fig. $4 \mathrm{c}$ shows the variation of the PPF index as a function of the interval $(\Delta t)$ for electrical spikes with a duration of $1 \mathrm{~ms}$. The PPF index decreases as $\Delta t$ increases. The dependence of the PPF index on $\Delta t$ is fitted using the following double-exponential equation [53]:

PPF index $=C_{1} \cdot \exp \left(-\frac{\Delta t}{\tau_{l}}\right)+C_{2} \cdot \exp \left(-\frac{\Delta \mathrm{t}}{\tau_{2}}\right)$,

where $C_{1}$ and $C_{2}$ are the initial facilitation magnitudes. $\tau_{1}$ and $\tau_{2}$ are the characteristic relaxation times for rapid decay and slow decay, respectively. We found that $\tau_{1}$ and $\tau_{2}$ are 11 and $47 \mathrm{~ms}$, respectively.

To mimic the synaptic function of LTP [66-73], we stimulated an electroluminescent synaptic device with multiple successive electrical spikes. Fig. $4 \mathrm{~d}$ shows the optical power of the device stimulated by from 2 to 30 spikes. It is clear that, as the number of electrical spikes increased from 2 to 20 , the optical power of the synaptic device increased. However, when the number of electrical spikes exceeded 20, the optical power of the device hardly changed. This means that the output of the electroluminescent synaptic device tends to be saturated after being stimulated by a large number of electrical spikes, consistent with the synaptic behavior of a biological neural system $[64,72]$. Note that the LTP delays of tens of milliseconds for our electroluminescent synaptic devices are shorter than the LTP decays of electrically stimulated synaptic devices [54]. However, they are comparable to the LTP decays of optically stimulated synaptic devices [65].

We finally demonstrate that the LTP of the electroluminescent synaptic device may be employed for simple image recognition, as shown in Fig. 4e-h. Since the number of squares recognized in the eagle image is associated with the ratio of the maximum optical power evoked by $i(i=2,5,10,20,30)$ electrical spikes $\left(P_{i}\right)$ to that evoked by 30 electrical spikes $\left(P_{30}\right)$, i.e., $P_{i} / P_{30}$, we see a clearer image as the number of electrical spikes increases.

\section{CONCLUSIONS}

The high hole mobility of $\mathrm{P} 3 \mathrm{HT}$ and its negligible absorption in the NIR region have enabled the incorporation of P3HT into NIR QLEDs as the HTL. A P3HT HTL significantly mitigates the carrier injection imbalance in NIR QLEDs. An interlayer of PFN can be added between the P3HT and QDs to further improve the performance of the NIR QLEDs. With this improved performance, these NIR QLEDs can be used as optoelectronic synaptic devices. They have important synaptic functionality, such as short- and long-term plasticity.

Received 13 April 2019; accepted 7 May 2019;

published online 31 May 2019

1 Zidan MA, Strachan JP, Lu WD. The future of electronics based on memristive systems. Nat Electron, 2018, 1: 22-29

2 Kuzum D, Yu S, Philip Wong HS. Synaptic electronics: Materials, devices and applications. Nanotechnology, 2013, 24: 382001

3 Abbott LF, Regehr WG. Synaptic computation. Nature, 2004, 431: 796-803

4 Prezioso M, Merrikh-Bayat F, Hoskins BD, et al. Training and operation of an integrated neuromorphic network based on metaloxide memristors. Nature, 2015, 521: 61-64

5 Tuma T, Pantazi A, Le Gallo M, et al. Stochastic phase-change neurons. Nat Nanotech, 2016, 11: 693-699

6 Jiang J, Guo J, Wan X, et al. 2D MoS 2 neuromorphic devices for brain-like computational systems. Small, 2017, 13: 1700933

7 Sangwan VK, Lee HS, Bergeron $\mathrm{H}$, et al. Multi-terminal memtransistors from polycrystalline monolayer molybdenum disulfide. Nature, 2018, 554: 500-504

8 Wang Z, Joshi S, Savel'ev SE, et al. Memristors with diffusive dynamics as synaptic emulators for neuromorphic computing. Nat Mater, 2017, 16: 101-108

9 van de Burgt Y, Lubberman E, Fuller EJ, et al. A non-volatile organic electrochemical device as a low-voltage artificial synapse for neuromorphic computing. Nat Mater, 2017, 16: 414-418

10 Shi Y, Liang X, Yuan B, et al. Electronic synapses made of layered two-dimensional materials. Nat Electron, 2018, 1: 458-465

11 Tian $\mathrm{H}, \mathrm{Mi} \mathrm{W}$, Zhao $\mathrm{H}$, et al. A novel artificial synapse with dual modes using bilayer graphene as the bottom electrode. Nanoscale, 2017, 9: 9275-9283

12 Zhu J, Yang Y, Jia R, et al. Ion gated synaptic transistors based on $2 \mathrm{D}$ van der Waals crystals with tunable diffusive dynamics. Adv Mater, 2018, 30: 1800195

13 Wang Y, Lv Z, Chen J, et al. Photonic synapses based on inorganic perovskite quantum dots for neuromorphic computing. Adv Mater, 2018, 30: 1802883

14 John RA, Liu F, Chien NA, et al. Synergistic gating of electro-ionophotoactive 2D chalcogenide neuristors: Coexistence of hebbian and homeostatic synaptic metaplasticity. Adv Mater, 2018, 30: 1800220

15 Cheng Z, Ríos C, Pernice WHP, et al. On-chip photonic synapse. Sci Adv, 2017, 3: e1700160

16 Akemann W, Song C, Mutoh H, et al. Route to genetically targeted optical electrophysiology: Development and applications of voltage-sensitive fluorescent proteins. Neurophotonics, 2015, 0210081

17 Qin S, Wang F, Liu Y, et al. A light-stimulated synaptic device based on graphene hybrid phototransistor. 2D Mater, 2017, 4: 035022

18 Wang S, Chen C, Yu Z, et al. A MoS 2 PTCDA hybrid heterojunction synapse with efficient photoelectric dual modulation and versatility. Adv Mater, 2019, 31: 1806227

19 Kim J, Lee HC, Kim KH, et al. Photon-triggered nanowire transistors. Nat Nanotech, 2017, 12: 963-968

20 Lee M, Lee W, Choi S, et al. Brain-inspired photonic neuromorphic devices using photodynamic amorphous oxide semiconductors and their persistent photoconductivity. Adv Mater, 2017, 29: 1700951 
21 Tan H, Liu G, Yang H, et al. Light-gated memristor with integrated logic and memory functions. ACS Nano, 2017, 11: 11298-11305

22 Tan $\mathrm{H}, \mathrm{Ni} \mathrm{Z}$, Peng W, et al. Broadband optoelectronic synaptic devices based on silicon nanocrystals for neuromorphic computing. Nano Energy, 2018, 52: 422-430

23 Zhao $\mathrm{S}$, Ni Z, Tan $\mathrm{H}$, et al. Electroluminescent synaptic devices with logic functions. Nano Energy, 2018, 54: 383-389

24 Peng HT, Nahmias MA, de Lima TF, et al. Neuromorphic photonic integrated circuits. IEEE J Sel Top Quantum Electron, 2018, 24: $1-15$

25 Gong X, Yang Z, Walters G, et al. Highly efficient quantum dot near-infrared light-emitting diodes. Nat Photon, 2016, 10: 253-257

26 Shen $\mathrm{H}$, Zheng $\mathrm{Y}$, Wang $\mathrm{H}$, et al. Highly efficient near-infrared light-emitting diodes by using type-II CdTe/CdSe core/shell quantum dots as a phosphor. Nanotechnology, 2013, 24: 475603

27 Dai X, Deng Y, Peng X, et al. Quantum-dot light-emitting diodes for large-area displays: Towards the dawn of commercialization. Adv Mater, 2017, 29: 1607022

28 Pan J, Quan LN, Zhao Y, et al. Highly efficient perovskite-quantum-dot light-emitting diodes by surface engineering. Adv Mater, 2016, 28: 8718-8725

29 Dai X, Zhang Z, Jin Y, et al. Solution-processed, high-performance light-emitting diodes based on quantum dots. Nature, 2014, 515: 96-99

30 Shi Z, Li Y, Zhang Y, et al. High-efficiency and air-stable perovskite quantum dots light-emitting diodes with an all-inorganic heterostructure. Nano Lett, 2017, 17: 313-321

31 Zhang $\mathrm{X}$, Lin $\mathrm{H}$, Huang $\mathrm{H}$, et al. Enhancing the brightness of cesium lead halide perovskite nanocrystal based green light-emitting devices through the interface engineering with perfluorinated ionomer. Nano Lett, 2016, 16: 1415-1420

32 Tanase C, Meijer EJ, Blom PWM, et al. Unification of the hole transport in polymeric field-effect transistors and light-emitting diodes. Phys Rev Lett, 2003, 91: 216601

33 Xiao J, Shi J, Liu $\mathrm{H}$, et al. Efficient $\mathrm{CH}_{3} \mathrm{NH}_{3} \mathrm{PbI}_{3}$ perovskite solar cells based on graphdiyne (GD)-modified P3HT hole-transporting material. Adv Energy Mater, 2015, 5: 1401943

34 Song B, He Y. Fluorescent silicon nanomaterials: From synthesis to functionalization and application. Nano Today, 2019

35 He Z, Zhong C, Su S, et al. Enhanced power-conversion efficiency in polymer solar cells using an inverted device structure. Nat Photon, 2012, 6: 591-595

36 Abbott LF, Nelson SB. Synaptic plasticity: Taming the beast. Nat Neurosci, 2000, 3: 1178-1183

37 Zhu LQ, Wan CJ, Guo LQ, et al. Artificial synapse network on inorganic proton conductor for neuromorphic systems. Nat Commun, 2014, 5: 3158-3165

38 Yu T, Wang F, Xu Y, et al. Graphene coupled with Silicon quantum dots for high-performance bulk-silicon-based Schottky-junction photodetectors. Adv Mater, 2016, 28: 4912-4919

39 Liu X, Zhang Y, Yu T, et al. Optimum quantum yield of the light emission from 2 to $10 \mathrm{~nm}$ hydrosilylated silicon quantum dots. Part Part Syst Charact, 2016, 33: 44-52

40 Li G, Shrotriya V, Huang J, et al. High-efficiency solution processable polymer photovoltaic cells by self-organization of polymer blends. Nat Mater, 2005, 4: 864-868

$41 \mathrm{Gu} \mathrm{W}$, Liu X, Pi X, et al. Silicon-quantum-dot light-emitting diodes with interlayer-enhanced hole transport. IEEE Photonics J, 2017, 9: 1-10

42 van Buuren T, Dinh LN, Chase LL, et al. Changes in the electronic properties of Si nanocrystals as a function of particle size. Phys Rev Lett, 1998, 80: 3803-3806

$43 \mathrm{Xu} \mathrm{T}$, Qiao Q. Conjugated polymer-inorganic semiconductor hybrid solar cells. Energy Environ Sci, 2011, 4: 2700-2720

44 Itskos G, Othonos A, Rauch T, et al. Optical properties of organic semiconductor blends with near-infrared quantum-dot sensitizers for light harvesting applications. Adv Energy Mater, 2011, 1: 802812

45 Shastry TA, Clark SC, Rowberg AJE, et al. Enhanced uniformity and area scaling in carbon nanotube-fullerene bulk-heterojunction solar cells enabled by solvent additives. Adv Energy Mater, 2016, 6: 1501466

46 Liu X, Zhao S, Gu W, et al. Light-emitting diodes based on colloidal silicon quantum dots with octyl and phenylpropyl ligands. ACS Appl Mater Interfaces, 2018, 10: 5959-5966

47 Huang F, Wu H, Cao Y. Water/alcohol soluble conjugated polymers as highly efficient electron transporting/injection layer in optoelectronic devices. Chem Soc Rev, 2010, 39: 2500-2521

48 Kim YH, Han TH, Cho H, et al. Polyethylene imine as an ideal interlayer for highly efficient inverted polymer light-emitting diodes. Adv Funct Mater, 2014, 24: 3808-3814

49 Zhao S, Liu X, Gu W, et al. $\mathrm{Al}_{2} \mathrm{O}_{3}$-interlayer-enhanced performance of all-inorganic silicon-quantum-dot near-infrared lightemitting diodes. IEEE Trans Electron Devices, 2018, 65: 577-583

50 Kwak J, Bae WK, Lee D, et al. Bright and efficient full-color colloidal quantum dot light-emitting diodes using an inverted device structure. Nano Lett, 2012, 12: 2362-2366

51 Bozyigit D, Yarema O, Wood V. Origins of low quantum efficiencies in quantum dot leds. Adv Funct Mater, 2013, 23: 30243029

52 Markram H. A history of spike-timing-dependent plasticity. Front Syn Neurosci, 2011, 3: 4

53 Liu YH, Zhu LQ, Feng P, et al. Freestanding artificial synapses based on laterally proton-coupled transistors on chitosan membranes. Adv Mater, 2015, 27: 5599-5604

54 Yan X, Zhao J, Liu S, et al. Memristor with Ag-cluster-doped $\mathrm{TiO}_{2}$ films as artificial synapse for neuroinspired computing. Adv Funct Mater, 2018, 28: 1705320

55 Feng P, Xu W, Yang Y, et al. Printed neuromorphic devices based on printed carbon nanotube thin-film transistors. Adv Funct Mater, 2017, 27: 1604447

56 Du C, Ma W, Chang T, et al. Biorealistic implementation of synaptic functions with oxide memristors through internal ionic dynamics. Adv Funct Mater, 2015, 25: 4290-4299

57 Chang T, Jo SH, Lu W. Short-term memory to long-term memory transition in a nanoscale memristor. ACS Nano, 2011, 5: 76697676

58 Yang CS, Shang DS, Liu N, et al. A synaptic transistor based on quasi-2D molybdenum oxide. Adv Mater, 2017, 29: 1700906

59 Fu YM, Wan CJ, Zhu LQ, et al. Hodgkin-huxley artificial synaptic membrane based on protonic/electronic hybrid neuromorphic transistors. Adv Biosys, 2018, 17001981

$60 \mathrm{Xu} \mathrm{W}$, Nguyen TL, Kim YT, et al. Ultrasensitive artificial synapse based on conjugated polyelectrolyte. Nano Energy, 2018, 48: 575581

61 Yang CS, Shang DS, Chai YS, et al. Electrochemical-reaction-induced synaptic plasticity in $\mathrm{MoO}_{x}$-based solid state electrochemical cells. Phys Chem Chem Phys, 2017, 19: 4190-4198

62 Tian H, Mi W, Wang XF, et al. Graphene dynamic synapse with modulatable plasticity. Nano Lett, 2015, 15: 8013-8019 
63 Nayak A, Ohno T, Tsuruoka T, et al. Controlling the synaptic plasticity of a $\mathrm{Cu}_{2} \mathrm{~S}$ gap-type atomic switch. Adv Funct Mater, 2012, 22: 3606-3613

64 Ohno T, Hasegawa T, Tsuruoka T, et al. Short-term plasticity and long-term potentiation mimicked in single inorganic synapses. Nat Mater, 2011, 10: 591-595

65 Jo SH, Chang T, Ebong I, et al. Nanoscale memristor device as synapse in neuromorphic systems. Nano Lett, 2010, 10: 1297-1301

66 Sarkar D, Tao J, Wang W, et al. Mimicking biological synaptic functionality with an indium phosphide synaptic device on silicon for scalable neuromorphic computing. ACS Nano, 2018, 12: 16561663

67 Yan X, Zhang L, Chen $\mathrm{H}$, et al. Graphene oxide quantum dots based memristors with progressive conduction tuning for artificial synaptic learning. Adv Funct Mater, 2018, 28: 1803728

68 Zhu X, Lu WD. Optogenetics-inspired tunable synaptic functions in memristors. ACS Nano, 2018, 12: 1242-1249

69 Yin J, Zeng F, Wan Q, et al. Adaptive crystallite kinetics in homogenous bilayer oxide memristor for emulating diverse synaptic plasticity. Adv Funct Mater, 2018, 28: 1706927

70 Jeong DS, Hwang CS. Nonvolatile memory materials for neuromorphic intelligent machines. Adv Mater, 2018, 30: 1704729

71 Choi S, Tan SH, Li Z, et al. SiGe epitaxial memory for neuromorphic computing with reproducible high performance based on engineered dislocations. Nat Mater, 2018, 17: 335-340

$72 \mathrm{Hu} \mathrm{L}, \mathrm{Fu} \mathrm{S}$, Chen Y, et al. Ultrasensitive memristive synapses based on lightly oxidized sulfide films. Adv Mater, 2017, 29: 1606927

73 Xiao Z, Huang J. Energy-efficient hybrid perovskite memristors and synaptic devices. Adv Electron Mater, 2016, 16001001

Acknowledgements The current work was mainly supported by the National Key Research and Development Program of China (2017YFA0205700) and the National Natural Science Foundation of China (NSFC, 61774133 and 6147409). Partial support from the NSFC for Innovative Research Groups (61721005) was also acknowledged.

Author contributions Pi X conceived the project. Zhao S, Wang Y, Jin $\mathrm{H}$, Huang $\mathrm{P}$, Wang $\mathrm{H}$ and Wang $\mathrm{K}$ performed the experiments. Huang $\mathrm{W}$ simulated the image recognition. Zhao $\mathrm{S}$ and $\mathrm{Pi} \mathrm{X}$ wrote the manuscript. All authors discussed the results and contributed to the writing.

Conflict of interest The authors declare that they have no conflict of interest.
Supplementary information online version of this paper

Supporting data are available in the

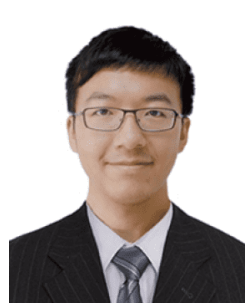

Shuangyi Zhao received his $\mathrm{PhD}$ degree in the School of Materials Science and Engineering at Zhejiang University, China, in 2018. He worked on the fabrication of silicon nanocrystals and their applications in optoelectronic devices such as solar cells, light-emitting devices, and synaptic devices.

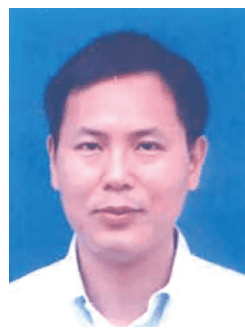

Xiaodong $\mathrm{Pi}$ received his $\mathrm{PhD}$ degree in the Department of Physics at the University of Bath, UK, in 2004. Further, he performed research in the Department of Engineering Physics at McMaster University and in the Department of Mechanical Engineering at the University of Minnesota, Twin Cities. He joined Zhejiang University as an associate professor in 2008 . Currently, he is a professor in the State Key Laboratory of Silicon Materials and the School of Materials Science and Engineering at Zhejiang University. His research mainly concerns silicon-based optoelectronic materials and devices.

\section{近红外量子点发光二极管用于模拟神经突触 可塑性}

赵双易 ${ }^{1}$, 王越, 黄稳, 金是 ${ }^{1}$, 黄沛文, 王虎 ${ }^{1}$, 王坤 ${ }^{1}$, 李东升 ${ }^{1}$, 徐明生 ${ }^{2}$, 杨德仁 ${ }^{1}$, 皮孝东

摘要 为了提高近红外发光二极管的性能, 我们利用聚3-已基噻吩 (P3HT) 空穴迁移率高和对近红外光没有吸收的特点, 将其作为器 件的空穴传输层. 实验发现, P3HT改善了基于硅量子点的近红外 发光二极管的空穴/电子传输不平衡的现象. 进一步地, 将聚 $[9,9$ 二(3'-( $N, N$-二甲胺基)丙基)-2,7-芴-交-2,7-(9,9-二辛基芴)](PFN)作 为中间层修饰 $\mathrm{P} 3 \mathrm{HT}$, 近红外硅量子点发光二极管的性能得到了更 大改善, 其外量子效率和功率效率分别达到了 $3.4 \%$ 和 $4.4 \%$. 性能改 善后的近红外硅量子点发光二极管可以用于模拟神经突触的可塑 性, 如短时程可塑性和长时程可塑性. 